\title{
Timing of surgical treatment for idiopathic normal pressure hydrocephalus: association between treatment delay and reduced short-term benefit
}

\author{
Sharif Vakili, MS, Dane Moran, BS, Alice Hung, BA, Benjamin D. Elder, MD, PhD, \\ Lee Jeon, Hugo Fialho, Eric W. Sankey, MD, Ignacio Jusué-Torres, MD, \\ C. Rory Goodwin, MD, PhD, Jennifer Lu, BA, Jamie Robison, BSN, and Daniele Rigamonti, MD \\ Department of Neurosurgery, The Johns Hopkins University School of Medicine, Baltimore, Maryland
}

\begin{abstract}
OBJECTIVE A growing body of evidence suggests that longer durations of preoperative symptoms may correlate with worse postoperative outcomes following cerebrospinal fluid (CSF) diversion for treatment of idiopathic normal pressure hydrocephalus (iNPH). The aim of this study is to determine whether the duration of preoperative symptoms alters postoperative outcomes in patients treated for iNPH.
\end{abstract}

METHODS The authors conducted a retrospective review of 393 cases of iNPH involving patients treated with ventriculoperitoneal (VP) shunting. The duration of symptoms prior to the operative intervention was recorded. The following outcome variables were assessed at baseline, 6 months postoperatively, and at last follow-up: gait performance, urinary continence, and cognition.

RESULTS The patients' median age at shunt placement was 74 years. Increased symptom duration was significantly associated with worse gait outcomes (relative risk (RR) 1.055 per year of symptoms, $p=0.037$ ), and an overall absence of improvement in any of the classic triad symptomology (RR 1.053 per year of symptoms, $p=0.033$ ) at 6 months postoperatively. Additionally, there were trends toward significance for symptom duration increasing the risk of having no 6-month postoperative improvement in urinary incontinence (RR 1.049 per year of symptoms, $p=0.069$ ) or cognitive symptoms (RR 1.051 per year of symptoms, $p=0.069$ ). However, no statistically significant differences were noted in these outcomes at last follow-up (median 31 months). Age stratification by decade revealed that prolonging symptom duration was significantly associated with lower Mini-Mental Status Examination scores in patients aged 60-70 years, and lack of cognitive improvement in patients aged 70-80 years.

CONCLUSIONS Patients with iNPH with longer duration of preoperative symptoms may not receive the same shortterm benefits of surgical intervention as patients with shorter duration of preoperative symptoms. However, with longer follow-up, the patients generally reached the same end point. Therefore, when managing patients with iNPH, it may take longer to see the benefits of CSF shunting when patients present with a longer duration of preoperative symptoms. http://thejns.org/doi/abs/10.3171/2016.6.FOCUS16146

KEY WORDS idiopathic normal pressure hydrocephalus; VP shunt; incontinence; gait abnormality; cognitive decline

$\mathrm{F}$ IRST identified in 1965,5 idiopathic normal pressure hydrocephalus (iNPH) is a syndrome of progressive neurological deterioration associated with ventricular enlargement but normal cerebrospinal fluid (CSF) pressure on lumbar puncture. ${ }^{8}$ Classically, the disease has been associated with a syndromic triad of gait disturbance, urinary incontinence, and cognitive decline, with gait disturbance and one other feature generally required for diagnosis. ${ }^{9,18,20}$ Generally, the diagnosis of iNPH is multifactorial and made through the assessment of clinical symptoms, imaging, CSF pressure measurements, and response to CSF drainage. Although the pathology underlying these cases is still unknown, iNPH remains one of the few causes of dementia and cognitive decline that is potentially reversible. ${ }^{2}$

The standard treatment for iNPH is surgical CSF diversion, most commonly through implantation of a ventriculoperitoneal (VP) shunt, to alleviate the clinical symptoms

ABBREVIATIONS CSF = cerebrospinal fluid; iNPH = idiopathic normal pressure hydrocephalus; $M$ MSE = Mini-Mental Status Examination; RR = relative risk; VP = ventriculoperitoneal. 
caused by excess intracranial fluid. ${ }^{16}$ The rate of successful shunt response in the treatment of iNPH has increased over the years. A 2001 meta-analysis of outcomes reported the treatment to have a $29 \%$ rate of significant improvement and a 6\% significant complication rate. ${ }^{6}$ More recent studies have revealed greater improvements, with $75 \%$ of patients $(n=132)$ seeing postoperative improvements within 24 months of surgery in a retrospective study, ${ }^{14} 68 \%$ of patients experiencing "very good" or "good" outcomes in a prospective study, ${ }^{15}$ and $69 \%-84 \%$ of patients seeing improvements by 1 year after surgery in a prospective multicenter study. ${ }^{23}$ However, given the significant number of patients who do not show improvement postoperatively, efforts have been taken to better identify potential responders to treatment and potential prognostic predictors that may be controlled. ${ }^{10}$

The duration of symptoms prior to surgical treatment is suggested to be a potential predictor of outcomes, with delayed treatment potentially decreasing treatment response. A retrospective study of 132 patients found duration of symptoms and gait as the primary symptom as the only independent predictors of outcomes in the surgical treatment of iNPH..${ }^{14}$ Similarly, a smaller prospective study involving 33 patients with iNPH found that those in whom surgical treatment was delayed for more than 3 months had significantly poorer final outcomes as evaluated by the modified Rankin Scale., ${ }^{1,17}$ However, the study attributed the worse outcomes to neurological deterioration prior to surgery, as the magnitude of change with surgery was comparable to that in patients without treatment delay.

Not all studies have resulted in the conclusion that delaying treatment ultimately leads to worse iNPH outcomes. The findings of one study suggested that worse outcomes observed in patients with delayed treatment may actually be a function of delayed response to treatment. ${ }^{7}$ In the open-label randomized trial of 93 patients undergoing lumbar-peritoneal surgery for iNPH, patients who had surgery postponed for 3 months had worse outcomes at 3 months after surgery compared with those who received surgery within 1 month of study enrollment: $65 \%$ in the immediate treatment group versus 5\% in the postponed treatment group saw an improvement of 1 point or more on the modified Rankin Scale. However, by 1 year after surgery, the difference in outcomes between the groups narrowed: $67 \%$ in the immediate treatment group versus $58 \%$ in the postponed treatment group saw an improvement of 1 point or more on the modified Rankin Scale. In another retrospective study involving 92 patients, improvement after CSF shunting did not exhibit any dependency on duration of symptoms. ${ }^{3}$

Therefore, the extent and ways in which delayed treatment influences postoperative clinical outcomes remain to be elucidated. Given a prevalence of as much as $1.4 \%$ in the elderly adult population, ${ }^{12,21}$ and $9 \%-14 \%$ in the assisted-living population, ${ }^{10,12}$ it is important to determine to what degree more aggressive identification of iNPH cases for early intervention may prevent morbidity and mortality. The objective of this study was to provide more clarity to these questions and to determine whether the duration of preoperative symptoms alters postoperative outcomes in patients treated for iNPH.

\section{Methods}

After approval was obtained from our institutional review board, a retrospective review was performed. All patients diagnosed with communicating idiopathic hydrocephalus and treated with VP shunts by the senior author (D.R.) at the Johns Hopkins Hospital between 1993 and 2013 were analyzed. Idiopathic NPH was diagnosed using standard clinical guidelines. ${ }^{9,18,20}$ Specifically, to more objectively identify patients with iNPH, the diagnosis was made by having at least 1 of the 3 cardinal iNPH symptoms of urinary incontinence, gait instability, and cognitive decline (with primarily at least gait instability) that improved following CSF removal during lumbar puncture (LP) and/ or lumbar drainage trials, with opening pressure of all included patients being less than $25 \mathrm{~cm} \mathrm{H}_{2} \mathrm{O}$. All patients underwent a CSF drainage trial prior to shunt placement. Additionally, the patients had normal morphology of the third ventricle on CT and/or MRI to exclude an obstructive etiology. Per diagnostic guidelines, ${ }^{18}$ patients younger than 40 years were excluded. Those with NPH after tumor surgery or NPH secondary to trauma or infection were also excluded.

Demographic information including baseline symptoms, age, sex, and race were recorded. In addition to clinical presentation, multiple markers were used to assess patient outcomes at six months following surgery and at last follow-up: MMSE, the modified Rankin Scale, the Barthel Index of Activities of Daily Living, the Wikkels $\varnothing$ score, the Timed Up-and-Go test, and the Tinetti balance assessment. Based on these results and subjective physician-documented assessments of clinical presentation, categorical changes in gait, urinary incontinence, and cognitive outcomes were also recorded 6 months following surgery and at last follow-up. Symptom changes were categorized as improvement, regression, or no change. An additional analysis was performed stratifying the patients by decade of age (40-50 years, 50-60 years, $60-70$ years, $70-80$ years, and $80+$ years).

\section{Statistics}

Data analysis was performed using Stata 12 (StataCorp, College Station, Texas, USA). Multinomial logistic regression was used to determine the relationship between duration of symptoms prior to surgery and improvement in symptoms. Simple linear regression was used to determine the relationship between duration of symptoms and performance on objective clinical studies. Multivariate models compared symptom improvement with age, sex, race, and prior treatment as well. All reported $\mathrm{p}$ values were 2 -sided and statistical significance was set at $\mathrm{p}<0.05$.

\section{Results}

A total of 393 patients were identified who met the criteria for inclusion in the study. Their median age at treatment was 74 years; $85 \%$ of patients were 65 years or older, $6 \%$ were between 60 and 65 years of age, and $9 \%$ were between ages 40 and 60 years of age. Patients predominantly presented with gait disturbance along with urinary incontinence and/or cognitive decline. In the series, $96.7 \%$ of the patients had gait disturbance, $85.5 \%$ presented with cognitive decline, and $88.0 \%$ presented with urinary inconti- 
TABLE 1. Summary of clinical and demographic characteristics of 393 patients with iNPH*

\begin{tabular}{lc}
\hline \multicolumn{1}{c}{ Characteristic } & Value \\
\hline Age at treatment, median & 74 yrs \\
\hline Sex & $224(57.0)$ \\
\hline Male & $169(43.0)$ \\
\hline Female & $355(90.3)$ \\
\hline Race & $21(5.3)$ \\
\hline Caucasian & $17(4.3)$ \\
\hline African American & $380(96.7)$ \\
\hline Other & $336(85.5)$ \\
\hline Baseline classic NPH symptoms & $346(88.0)$ \\
\hline Gait disturbance & 28 mos \\
\hline Urinary incontinence & 31 mos \\
\hline Cognitive decline & 0.367 \\
\hline Preop symptom duration, median & \\
\hline Follow-up duration, median & \\
\hline Preop Evans Index, median $(\mathrm{n}=149)$ &
\end{tabular}

* Values represent number of patients (\%) unless otherwise indicated.

nence (Table 1). Additionally, the baseline symptomatology was similar among patients with different symptom durations (Figs. 1-5). The median preoperative duration of symptoms was 28 months, the median follow-up duration was 31 months, and the median preoperative Evans index was 0.367 (Table 1). Of all measured outcomes, statistically significant associations with preoperative symptom duration were observed among categorical clinical outcomes.
Risk of worse gait outcomes 6 months postoperatively was noted with prolonged symptom duration: for every year of increased symptom duration, there was a $5.5 \%$ increased risk of having worse gait outcomes after surgery (relative risk [RR] 1.055, $\mathrm{p}=0.037$ ). Increased symptom duration was also associated with risk of failure to improving in any one of the classic hydrocephalus symptoms (gait impairment, urinary incontinence, and cognitive decline): for every year of increased symptom duration, there was a $5.3 \%$ increased risk of having no improvement in gait, urinary incontinence, or cognitive outcomes 6 months postoperatively ( $R R$ 1.053, $\mathrm{p}=0.033$ ). Trends were observed between increased symptom duration increasing the risk of having no 6-month postoperative improvement in urinary incontinence (RR 1.049 per year, $\mathrm{p}=0.069)$ or cognitive symptoms (RR 1.051 per year, $\mathrm{p}=0.069$ ). However, when results were stratified by decade, a significant association was seen between symptom duration and risk of having no 6-month postoperative improvement in cognition (RR 1.121 per year, $\mathrm{p}=0.027$ ) among patients $70-80$ years of age.

No statistical significance was noted with any of these assessments at last follow-up, and no other variables, including age, sex, race, or prior treatment substantially contributed in a multivariate model. Additionally, no statistically significant associations were found between preoperative symptom duration and any quantitative measurements of outcome such as the MMSE, the modified Rankin Scale, the Barthel Index, the Wikkels $\varnothing$ score, the Timed Up-and-Go test, and the Tinetti balance assessment. Decreased statistical power was seen with these analyses, as not all patients had all of the assessments due to evolutions

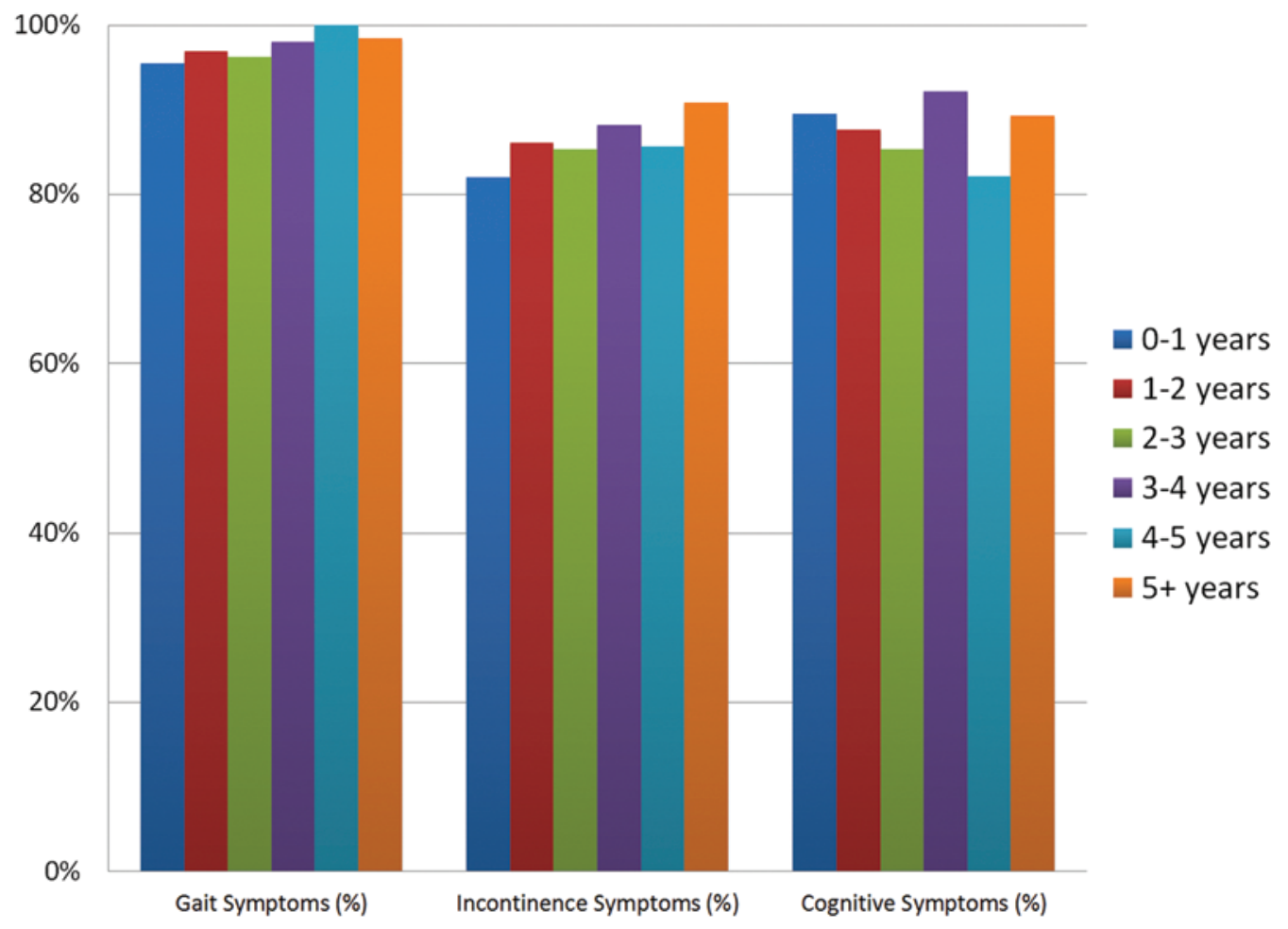

FIG. 1. Baseline symptoms versus symptom duration. There were no statistically significant differences in baseline symptoms among patients with different symptom durations. 


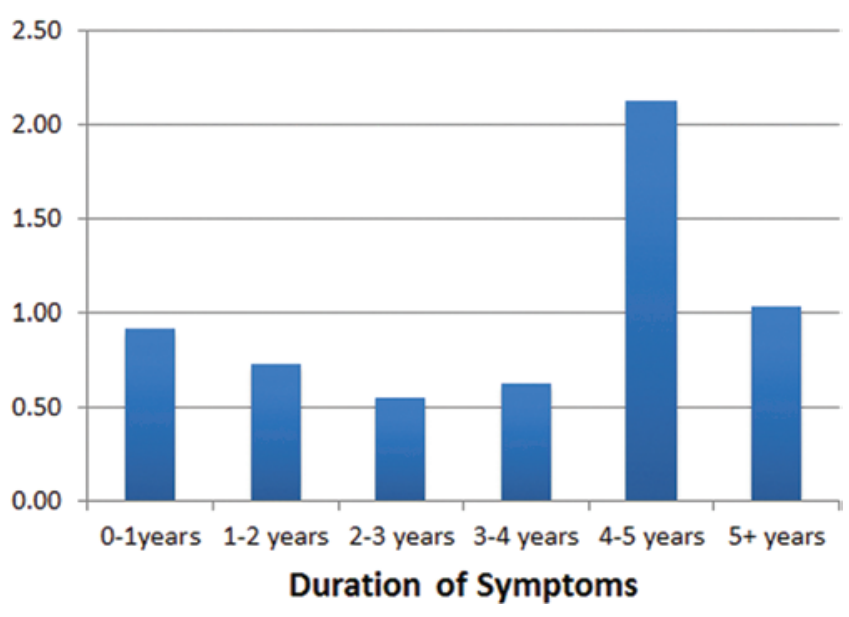

FIG. 2. Odds of no improvement in urinary incontinence at 6 months versus symptom duration. Although not statistically significant, there was a trend between preoperative symptom duration and increased risk of not having improvement in urinary incontinence 6 months after surgery (RR 1.049, $p=0.069$ )

in medical practice. However, when results were stratified by decade, there was a significant association between prolonged symptom duration and lower MMSE scores. For patients between 60-70 years of age, each month of symptoms was associated with a decrease of 0.101 point in MMSE score improvement 6 months postoperatively $\left(\mathrm{R}^{2}=\right.$ $0.194, \mathrm{~F}(1,24)=4.83, \mathrm{p}=0.040)$.

\section{Discussion}

To the best of our knowledge, this study is the first to provide an analysis of the effects of preoperative symptom duration on each of the 3 cardinal iNPH symptoms. Its findings can help determine which outcomes could be expected when counseling patients with iNPH for surgery depending on their specific preoperative symptom duration. Adding to the discourse on this topic initiated by our group ${ }^{14}$ and corroborated by others, ${ }^{1,3,7}$ this study supports the importance of prompt diagnosis and treatment of iNPH. Interestingly, the results suggest that patients with iNPH with longer duration of preoperative symptoms may not receive the same short-term benefits of surgical intervention as patients with shorter duration of preoperative symptoms, but with longer follow-up, the patients generally reached the same end point. Therefore, when managing patients with iNPH, it may take longer to see the benefits of CSF shunting when patients present with a longer duration of preoperative symptoms.

Age stratification revealed that prolonging symptom duration is significantly associated with lower MMSE scores in patients 60-70 years of age and lack of cognitive improvement in patients $70-80$ years of age. This may suggest that capturing improvements in cognition may be more challenging in more elderly patients when cognitive improvements may be more subtle. However, all of these differences in outcomes were seen only at 6 months after treatment and were not captured at the last follow-up visit after treatment.

There may be some limitation to our conclusions based on the selection of age $>40$ years in the inclusion criteria. There is heterogeneity in the literature regarding the age range for the diagnosis of iNPH. According to the international guidelines, ${ }^{18,24}$ an age threshold of 40 years

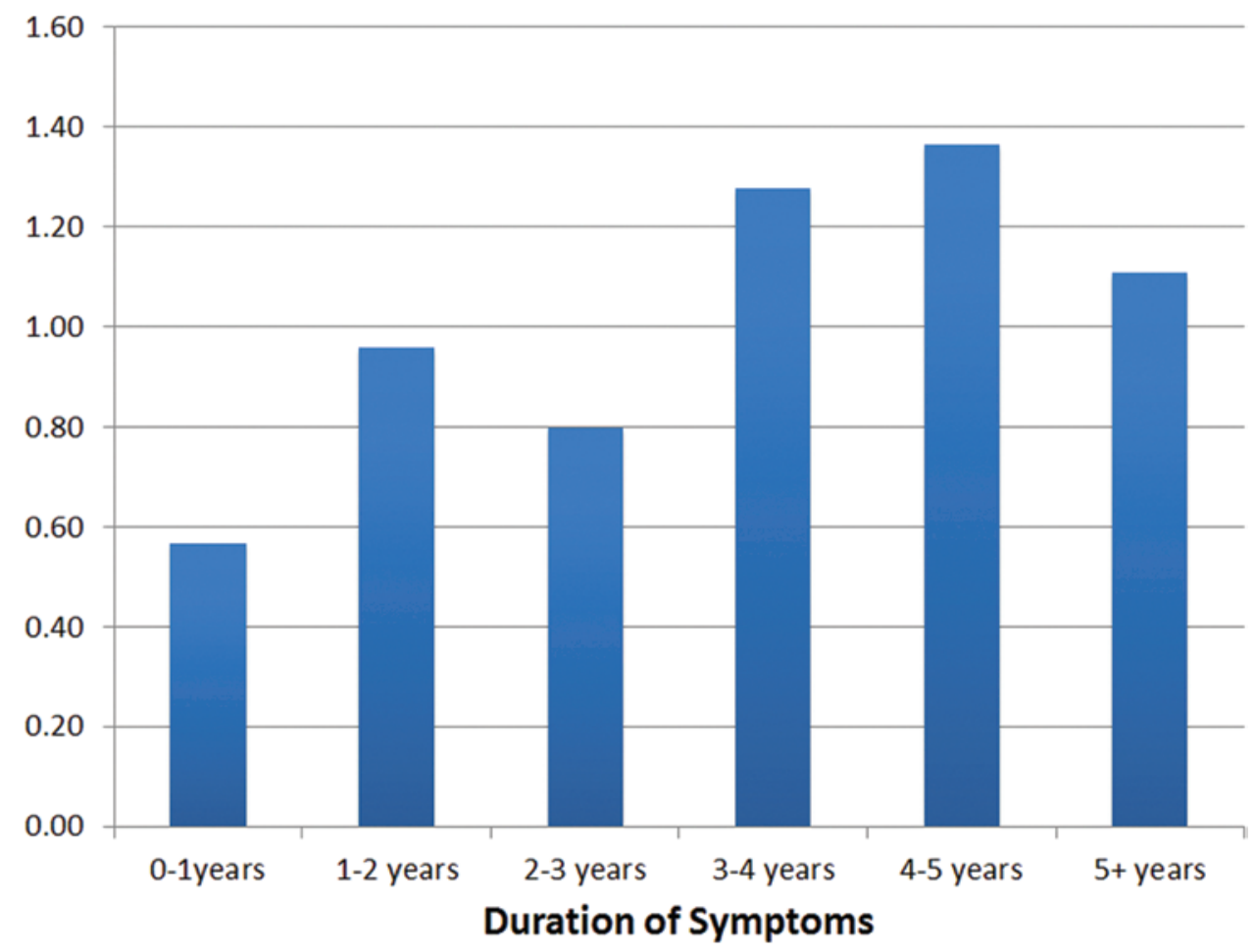

FIG. 3. Odds of no improvement in cognition at 6 months versus symptom duration. Although not statistically significant, there was a trend between preoperative symptom duration and increased risk of not having cognitive improvement 6 months after surgery (RR 1.051, $p=0.069$ ). 


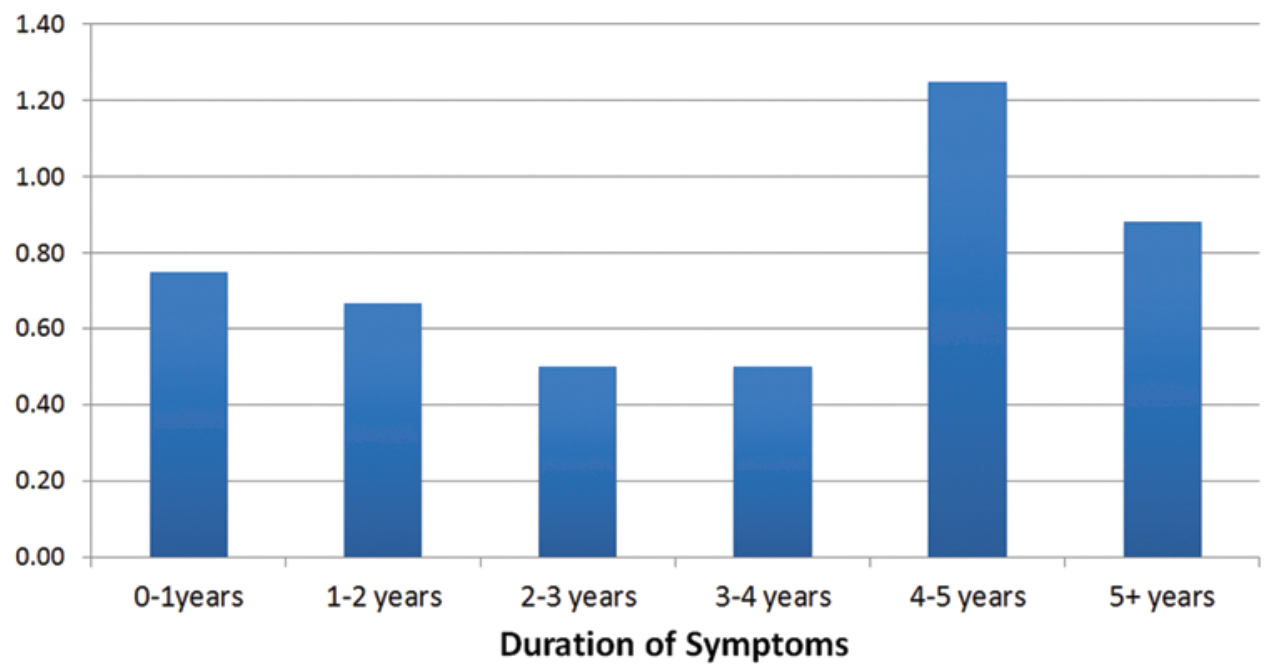

FIG. 4. Odds of worse gait outcomes versus symptom duration. There was an association between increased risk of negative gait outcomes 6 months after surgery and increased symptom duration (RR 1.055, $p=0.037$ ).

was used. In the 2012 Japanese iNPH guidelines, ${ }^{16}$ an age threshold of 60 years was used, and the guideline authors found that studies uncommonly found patients with an iNPH diagnosis prior to age 60 . However, some studies on iNPH have included patients younger than 60 years of age, ${ }^{4,11}$ and one study include patients as young as 34 years. ${ }^{4}$ As the vast majority of patients in our study were treated for iNPH prior to the formulation of the 2012 Japanese iNPH guidelines, ${ }^{16}$ we elected to use the international iNPH guidelines,${ }^{18}$ which identified an age criterion of 40 years or older for the diagnosis of iNPH. It is possible that this lower age range could lead to inclusion of patients with arrested congenital hydrocephalus, such as long-standing overt ventriculomegaly; however, the preoperative imaging requirement for normal third ventricular morphology and nonobstructive etiology should have decreased this risk. Ideally, it would be beneficial to stratify the patients by age $40-60$ years versus age $>60$ years, but there was not an adequate sample size to power this stratified analysis. Additionally, the median age of our patients at treatment was still 74 years, which is similar to that identified in the 2012 Japanese iNPH guidelines. ${ }^{16}$

Although these results show improved outcomes with more prompt treatment, the differences between the groups treated earlier or later were lost over time, with both groups demonstrating improvement with longer follow-up. This has also been seen in prior studies. ${ }^{6}$ One reason for this trend may be that as patients with iNPH grow older,

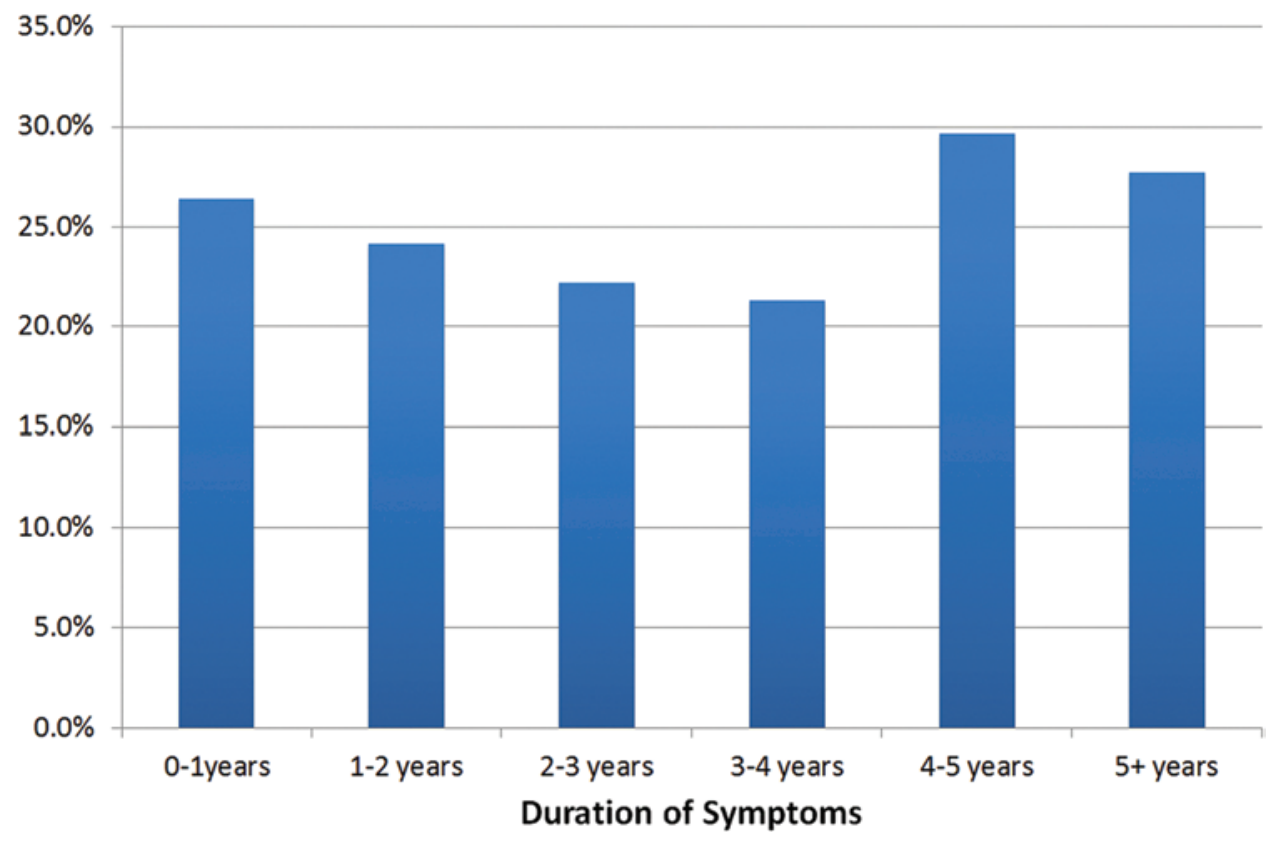

FIG. 5. Percentage of patients without improvement in any of the triad symptoms 6 months after surgery. There was an association between preoperative symptom duration and increased risk of not having improvement in any one of the triad iNPH symptoms (RR $1.053, p=0.033)$. 
other comorbidities may influence treatment outcomes. In our data, however, additional confounders may have also influenced this result. An important confounder is the heterogeneity in last follow-up time points. For some patients, the last follow-up visit was very close to surgery while others had a last follow-up visit much later in the course of their disease. The variation in the timing of the last followup visit can potentially mask trends. It is also possible that the lack of improvement in patients with delayed shunting may just represent further progression of the disease prior to treatment. Further studies assessing outcomes several years postoperatively would be beneficial in confirming whether the risks of delaying treatment are seen later in the disease course.

Another confounder of this study is that worse outcomes with delayed treatment may be functions of measuring outcomes later in the disease course, partly as a result of the insensitivity of our current diagnostic tests for iNPH. Patients who received delayed treatment may have ultimately ended up with the same outcome further along their presentation even had they received prompt treatment. Similarly, it is possible that since an accurate diagnosis of iNPH can be challenging, patients who presented with longer symptom duration may have had a less certain diagnosis. Nevertheless, in this study, standardized diagnostic assessments were used to identify patients with iNPH, and as shown in Fig. 1, patients with different symptom durations had similar baseline symptoms, so the certainty of iNPH diagnosis was presumably similar among the patients with different symptom durations. Furthermore, predicting how this confounder may have influenced the data can be difficult because iNPH does not progress the same way in all cases: without treatment, patients' condition can worsen, stagnate, or even improve..$^{22}$ Additionally, a patient's condition may worsen because of comorbidities unrelated to their iNPH.

Finally, another inherent weakness of any study looking at symptom duration is that determining accurate symptom durations can be difficult. As previous studies have suggested, patients are often unaware that they have symptoms of iNPH. ${ }^{10,21}$ Future large population-wide prospective studies may be able to remedy some of this if a population is followed with regular, standardized assessments for iNPH, although often the disease is misdiagnosed. ${ }^{19}$

While this study confirms the importance of prompt treatment for iNPH, future studies, particularly prospective multicenter registries and randomized trials, are needed to look at outcomes in patients with delayed treatment compared with patients who received no treatment and patients who received prompt treatment. Such a study would be able to correct for variabilities in iNPH disease presentation and disease course by comparing patients at the same stage of disease. Such studies could also benefit from closer analyses of quantitative measures of clinical outcomes, such as the MMSE, the modified Rankin Scale, the Barthel Index of Activities of Daily Living, the Wikkels $\varnothing$ scores, the Timed Up-and-Go test, and the Tinetti balance assessment. In our study, no statistically significant associations were found with these measures independently, probably because not all patients in this study had the tests performed as record-taking practices evolved, leading to decreased statistical power. Studies capturing associa- tions with these quantitative clinical assessments would be able to provide granularity to these results.

Nevertheless, the results of this study are important in guiding practice toward more prompt identification and treatment of iNPH for more rapid symptom improvement. Although some may believe that the efficacy of surgery for iNPH may still be uncertain,,$^{13}$ the findings of this study reaffirm that CSF diversion surgery helps with iNPH treatment, since even with delayed treatment, by every outcome measure, improvements were seen 6 months after surgery. Indeed, the odds of no improvement in cognition, gait, and urinary incontinence were all less than 1 when preoperative symptom duration was less than 4 years.

\section{Conclusions}

This study reinforces the notion that patients with longstanding preoperative symptoms may not receive the same benefits of surgical intervention as patients with shorter duration of preoperative symptoms. However, with longer follow-up, the patients generally reached the same end point. Therefore, when treating patients with iNPH, it may take longer to see the benefits of CSF shunting when patients present with a longer duration of preoperative symptoms.

\section{Acknowledgments}

We acknowledge research support from the Salisbury Family Foundation and the Swenson Foundation.

\section{References}

1. Andrén K, Wikkels $\varnothing$ C, Tisell M, Hellström P: Natural course of idiopathic normal pressure hydrocephalus. J Neurol Neurosurg Psychiatry 85:806-810, 2014

2. Conn HO: Normal pressure hydrocephalus: new complications and concepts. Pract Neurol 7:252-258, 2007

3. Czosnyka Z, Owler B, Keong N, Santarius T, Baledent O, Pickard JD, et al: Impact of duration of symptoms on CSF dynamics in idiopathic normal pressure hydrocephalus. Acta Neurol Scand 123:414-418, 2011

4. Eide PK: Intracranial pressure parameters in idiopathic normal pressure hydrocephalus patients treated with ventriculoperitoneal shunts. Acta Neurochir (Wien) 148:21-29, 2006

5. Hakim S, Adams RD: The special clinical problem of symptomatic hydrocephalus with normal cerebrospinal fluid pressure. Observations on cerebrospinal fluid hydrodynamics. J Neurol Sci 2:307-327, 1965

6. Hebb AO, Cusimano MD: Idiopathic normal pressure hydrocephalus: a systematic review of diagnosis and outcome. Neurosurgery 49:1166-1186, 2001

7. Kazui H, Miyajima M, Mori E, Ishikawa M: Lumboperitoneal shunt surgery for idiopathic normal pressure hydrocephalus (SINPHONI-2): an open-label randomised trial. Lancet Neurol 14:585-594, 2015

8. Klassen BT, Ahlskog JE: Normal pressure hydrocephalus: how often does the diagnosis hold water? Neurology 77:1119-1125, 2011

9. Krauss JK, Halve B: Normal pressure hydrocephalus: survey on contemporary diagnostic algorithms and therapeutic decision-making in clinical practice. Acta Neurochir (Wien) 146:379-388, 2004

10. Marmarou A, Young HF, Aygok GA: Estimated incidence of normal pressure hydrocephalus and shunt outcome in patients residing in assisted-living and extended-care facilities. Neurosurg Focus 22(4):E1, 2007 
11. Marmarou A, Young HF, Aygok GA, Sawauchi S, Tsuji O, Yamamoto T, et al: Diagnosis and management of idiopathic normal-pressure hydrocephalus: a prospective study in 151 patients. J Neurosurg 102:987-997, 2005

12. Martín-Láez R, Caballero-Arzapalo H, López-Menéndez LA, Arango-Lasprilla JC, Vázquez-Barquero A: Epidemiology of idiopathic normal pressure hydrocephalus: a systematic review of the literature. World Neurosurg 84:2002-2009, 2015

13. McGirr A, Mohammed S, Kurlan R, Cusimano MD: Clinical equipoise in idiopathic normal pressure hydrocephalus: a survey of physicians on the need for randomized controlled trials assessing the efficacy of cerebrospinal fluid diversion. J Neurol Sci 333:13-18, 2013

14. McGirt MJ, Woodworth G, Coon AL, Thomas G, Williams MA, Rigamonti D: Diagnosis, treatment, and analysis of long-term outcomes in idiopathic normal-pressure hydrocephalus. Neurosurgery 57:699-705, 2005

15. Meier U, Kiefer M, Neumann U, Lemcke J: On the optimal opening pressure of hydrostatic valves in cases of idiopathic normal-pressure hydrocephalus: a prospective randomized study with 123 patients. Acta Neurochir Suppl 96:358-363, 2006

16. Mori E, Ishikawa M, Kato T, Kazui H, Miyake H, Miyajima $\mathrm{M}$, et al: Guidelines for management of idiopathic normal pressure hydrocephalus: second edition. Neurol Med Chir (Tokyo) 52:775-809, 2012

17. Rankin J: Cerebral vascular accidents in patients over the age of 60. II. Prognosis. Scott Med J 2:200-215, 1957

18. Relkin N, Marmarou A, Klinge P, Bergsneider M, Black PM: Diagnosing idiopathic normal-pressure hydrocephalus. Neurosurgery 57 (3 Suppl):S4-S16, ii-v, 2005

19. Schirinzi T, Sancesario GM, Ialongo C, Imbriani P, Madeo $\mathrm{G}$, Toniolo S, et al: A clinical and biochemical analysis in the differential diagnosis of idiopathic normal pressure hydrocephalus. Front Neurol 6:86, 2015

20. Shprecher D, Schwalb J, Kurlan R: Normal pressure hydrocephalus: diagnosis and treatment. Curr Neurol Neurosci Rep 8:371-376, 2008

21. Tanaka N, Yamaguchi S, Ishikawa H, Ishii H, Meguro K: Prevalence of possible idiopathic normal-pressure hydro- cephalus in Japan: the Osaki-Tajiri project. Neuroepidemiology 32:171-175, 2009

22. Toma AK, Stapleton S, Papadopoulos MC, Kitchen ND, Watkins LD: Natural history of idiopathic normal-pressure hydrocephalus. Neurosurg Rev 34:433-439, 2011

23. Wikkels $\varnothing$ C, Hellström P, Klinge PM, Tans JT: The European iNPH Multicentre Study on the predictive values of resistance to CSF outflow and the CSF Tap Test in patients with idiopathic normal pressure hydrocephalus. J Neurol Neurosurg Psychiatry 84:562-568, 2013

24. Williams MA, Relkin NR: Diagnosis and management of idiopathic normal-pressure hydrocephalus. Neurol Clin Pract 3:375-385, 2013

\section{Disclosures}

The authors report no conflict of interest concerning the materials or methods used in this study or the findings specified in this paper. This manuscript represents the views of the authors and is not meant to represent FDA's views or policies.

\section{Author Contributions}

Conception and design: Elder, Vakili, Moran, Hung, Jusué-Torres, Rigamonti. Acquisition of data: Vakili, Moran, Hung, Sankey. Analysis and interpretation of data: Vakili, Moran, Hung, Jeon, Fialho, Sankey. Drafting the article: Vakili, Moran, Hung, Jeon, Fialho. Critically revising the article: Elder, Sankey, Jusué-Torres, Goodwin, Rigamonti. Reviewed submitted version of manuscript: Elder, Vakili, Jusué-Torres, Goodwin, Lu, Robinson, Rigamonti. Approved the final version of the manuscript on behalf of all authors: Elder. Statistical analysis: Vakili, Moran, Hung, Jusué-Torres. Administrative/technical/material support: Fialho, Jusué-Torres, Goodwin, Lu, Robinson. Study supervision: Elder, Goodwin, Rigamonti.

\section{Correspondence}

Benjamin D. Elder, Department of Neurosurgery, The Johns Hopkins University School of Medicine, 1800 Orleans St., Baltimore, MD 21287. email: belder4@jhmi.edu. 